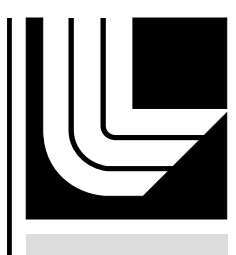

LAWRENCE LIVERMORE N A T IO N A L LABORATORY

\title{
UCRL-JRNL-227094
}

\section{APTASENSORS FOR BIOSECURITY APPLICATIONS}

Nicholas Fischer, Ted Tarasow, Jeffrey Tok

January 5, 2007

Current Opinion in Chemical Biology 
This document was prepared as an account of work sponsored by an agency of the United States Government. Neither the United States Government nor the University of California nor any of their employees, makes any warranty, express or implied, or assumes any legal liability or responsibility for the accuracy, completeness, or usefulness of any information, apparatus, product, or process disclosed, or represents that its use would not infringe privately owned rights. Reference herein to any specific commercial product, process, or service by trade name, trademark, manufacturer, or otherwise, does not necessarily constitute or imply its endorsement, recommendation, or favoring by the United States Government or the University of California. The views and opinions of authors expressed herein do not necessarily state or reflect those of the United States Government or the University of California, and shall not be used for advertising or product endorsement purposes. 


\title{
APTASENSORS FOR BIOSECURITY APPLICATIONS
}

\author{
Nicholas O. Fischer, Theodore M. Tarasow and Jeffrey B.-H. Tok ${ }^{*}$ \\ BioSecurity and NanoSciences Laboratory \\ Chemistry, Materials \& Life Sciences Directorate \\ Lawrence Livermore National Laboratory \\ Livermore, CA 94551 USA
}

SUMMARY: Nucleic acid aptamers have found steadily increased utility and application steadily over the last decade. In particular, aptamers have been touted as a valuable complement to and in some cases replacement for antibodies due to their structural and functional robustness as well as their ease in generation and synthesis. They are thus attractive for biosecurity applications, e.g. pathogen detection, and are especially well suited since their in vitro generation process does not require infection of any host systems. Herein we provide a brief overview of the aptamers generated against biopathogens over the last few years. In addition, a few recently described detection platforms using aptamers (aptasensors) and potentially suitable for biosecurity applications will be discussed.

\section{INTRODUCTION}

Biosecurity refers to the policies and procedures designed to prevent the deliberate theft or diversion of deadly pathogens and toxins for malicious or criminal purposes. While the goal is clearly to prevent any such criminal act, crucial in an effective response to a bioterrorism attack will be the swiftness and accuracy in both medical and environmental detection and characterization of the threat.[1,2] Even though great strides have been achieved in the environmental surveillance of potential biothreat agents, technologies needed for fabricating efficient, portable, fieldable and highly robust biopathogen sensors largely remain.[3,4]•• Many of the existing pathogen-sensing devices utilize antibodies as molecular recognition elements that mediate some signal transduction indicating the presence of the pathogen. However antibodies are limited by their structural robustness when exposed to varying temperatures and humidity and there remains an unfulfilled need in generating and producing all

\footnotetext{
${ }^{*}$ To whom correspondence should be addressed. E-mail: tok2@1lnl.gov; Phone : 925-423-1549, Fax: 925-423-3570.

Abbreviations: nucleotide, nt; molecular recognition element, MRE; surface plasmon resonance, SPR; hemagglutinin, HA; nitrocellulose, NC; gold, Au; nanoparticles, NP; quantum dot, QD, prostate-specific antigen (PSA); platelet-derived growth factor (PDGF); capillary electrophoresis, CE.
} 
of the antibodies required against the myriad types of biothreats and the especially high affinity monoclonal antibodies.

With these limitations, nucleic acid aptamers (target binding sequences generated through reiterative partitioning and selection process known as SELEX [5,6]) have recently been touted as valuable complements and in some cases potential antibody replacements due to their structural robustness and their ease in generation, synthesis and modification.[7,8] In addition, nucleic acid aptamers have become especially attractive as their generation process is not in vivo, but rather in vitro and no exposure to a host system is required.

Over the years, nucleic acid aptamers have been generated against a range of targets and adapted for numerous chemical or biological sensing systems. It is not the intent of this review to provide an exhaustive compilation of all these entries. Rather, a few recent examples that highlight the applications and utilities of the nucleic acid aptamers toward biosecurity applications will be described.

\section{APTAMERS AS BIOTHREAT PATHOGEN SENSING LIGANDS}

Even with their efficient generation process, aptamers are significantly less developed compared to antibodies against biothreat-relevant agents. In recent years, there have been intermittent reports in which aptamers have been generated against potential biothreat agents. Both the Center for Disease Control (CDC; www.bt.cdc.gov/bioterrorism/) and the National Institute of Allergy and Infectious Disease (NIAID; www3.niaid.nih.gov/Biodefense/PDF/cat.pdf) have published a list of priority pathogens (Category A to C), with Cat-A being the highest priority in term of their threats to national health and security. A brief survey of the biothreat agents and the availability of aptamers that have been generated against these targets follows.

Bacillus anthracis spores (Cat-A pathogen). The anthrax (bacillus anthracis) spore incident after 9/11 illustrates the simple fact that microorganisms can be disseminated to not only cause infection, but perhaps more damagingly, inflict fear and panic in the general public. Thus, increasing attention and resources have been since focused for rapid pathogen detection and characterization. B. anthracis are gram positive, soil-borne bacteria, endemic to many regions in the US and around the world with $\sim 90$ bacillus strains having been reported to date. Most of these strains do not pose any great threat however, among, the most virulent strain is the Ames strain (used in the postal attack) while the Vollum strain has been deemed most suitable for bioweaponization. As the bacteria are able to reside dormant in the soil and other media as endospores (spores), they can survive for a long period under varying environmental conditions. When inhaled and/or accidentally consumed, the bacteria can rapidly multiply in their host animal, ultimately killing it. It is also noted that as they are able to replicate in carcasses, humans can also get infected when they are exposed to the infected animal tissues. 
In an attempt to develop alternatives to antibody affinity reagents, ssDNA aptamers generated against autoclaved bacillus spores derived from stern strain were first described by Bruno and Kiel in the late 1990s.[9] The authors utilized centrifugation for partitioning the unbound DNA sequences from the DNA-spore complexes. Bruno and Kiel used both an aptamer-magnetic bead-electrochemiluminescence sandwich assay and a colorimetric assay to demonstrate binding to the anthrax spore target and established a wide dynamic range from $<10->6 \times 10^{6}$ anthrax spores. However, the spore-binding DNA aptamer sequences and their respective affinities were not reported limiting the utility of this report to serve as the basis of optimizing these aptamers or applying them to other sensing platforms.

A similar set of experiments was recently undertaken by Yang and coworkers, in which DNA aptamers were generated against spores of $b$. anthracis vaccine strain A 16R.[10] A total of 15 selection rounds were performed with a starting ssDNA library that contains a $35 \mathrm{nt}$ randomized region. DNA that was bound to the bacterial spores was separated by centrifugation and amplified via PCR. At the end of the selection cycles, the remaining DNA was cloned and sequenced. Among the obtained sequences, one full length 78nt aptamer (Apt1) was resynthesized and employed as a molecular recognition element (MRE) in a membrane-based hybrid sandwich assay. The assay is essentially comprised of an anti-spore antibody and labeled aptamer. Via this assay, it was observed that a ratio of $16 \mu \mathrm{g}$ of Apt1 was able to detect $\sim 4 \times 10^{7}$ spores, with the dynamic range from $4 \times 10^{7}$ to $4 \times 10^{4}$. Upon further minimization studies, it was realized that the aptamer, Apt2 (32nt), had the best binding affinities compared to other sequences studied. Upon further minimization to Apt3 (27nt), the binding effect was almost abolished, showing that the seemingly unstructured ssDNA tail was also important for spore binding. The secondary structures for all the studied anti-spore aptamers are predicted by the Mfold program,[11] and are shown in Fig. 1A.

Influenza Virus (Cat-C pathogen). Influenza, commonly known as flu, is caused by a RNAbased virus that belongs to the Orthomyxoviridae family. The virus can easily be transmitted via bodily fluids or through the air from infected individuals. In the case of animals or birds, transmissions usually occur through their droppings. Influenza has resulted in widespread disease and considerable death for centuries, with pandemics occurring in the last century approximately every 20 to 30 years Of recent concern is a deadly strain of avian influenza, H5N1, that is highly lethal to humans but because it is not easily transmitted between humans has only resulted in $\sim 100$ human fatalities.[12] However, a mutation to this $\mathrm{H} 5 \mathrm{~N} 1$ stain could result in a strain that is more easily transmitted among human hosts thus surveillance of the existing disease as well as indications of any mutated forms is of high priority..

Hemagglutinin (HA) and neuraminidase (NA) are two major glycoproteins present on the surface of the influenza virus envelope. Through HA, the virus is able to bind to sialic acid-modified receptors on host cells, and thus initiate viral infection by facilitating viral entry. Flu surveillance makes it essential 
that the various subtypes and even closely related viral strains can be accurately determined in a timely manner. Complicating this task is the fact that the HA and NA proteins on the surface of the influenza virus are able to undergo rapid mutation from generation to generation,. These mutations have also made the vaccine generation process against the virus much more difficult.

In the first of a series of approaches to generating aptamers against the HA protein, Kumar and co-workers utilized the surface plasmon resonance (SPR) technique to isolate RNA aptamers against purified HA of human A influenza virus, subtype H3N2.[13] This novel approach conveniently allowed for both the simultaneous monitoring and binding of the aptamers to the HA target. In a subsequent report, the same group selected RNA aptamers against whole H3N2 virus particles. The anti-HA RNA aptamers obtained displayed remarkable specificity as they were shown to differentiate HA proteins from closely related H3N2 strains.[14]•• After analyzing the sequences obtained after 10 selection cycles (partitioning via nitrocellulose filters), the highest HA binding aptamer (Apt4, structure shown in Fig. 1B) showed a $K_{d}$ of $\sim 188 \mathrm{pM}$ as measured via SPR, which was $\sim 15$-fold higher than the existing anti-HA monoclonal antibody $\left(\mathrm{K}_{\mathrm{d}}\right.$ of $\left.\sim 2.9 \mathrm{nM}\right)$. From sequence analysis of multiple clones a consensus sequences

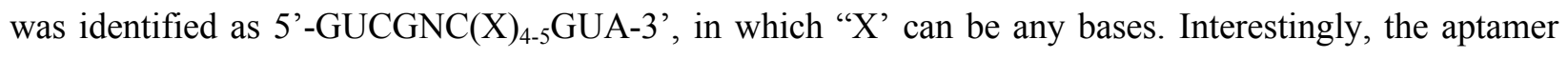
was observed to be able to inhibit the HA-mediated membrane fusion process, which greatly underscores its potential application as anti-influenza therapeutics. In another separate report, the same group has also utilized the same selection approach to generate RNA aptamers against the HA of human influenza B (a total of 9 selection cycles performed).[15] Among the obtained sequences, one of the aptamer was observed to demonstrate high selectivity as it is able to differentiate between the HA from influenza A and $B$ viruses. Again via SPR, its $K_{d}$ was measured to be $\sim 720 \mathrm{pM}$ and, similar to human influenza $A$, the generated RNA aptamer is observed to be able to inhibit HA-mediated membrane fusion process for human influenza B.

Instead of using RNA aptamers, Arnon and co-workers have shown that DNA aptamers can also be efficiently evolved against a specific domain of HA from influenza A virus.[16] By simply expressing a specific His-tagged oligosaccharide receptor domain (amino acids 91-261) of the HA and subjecting to the SELEX process via affinity partitioning approach, selected anti-HA ssDNA aptamer (Apt5, structure shown in Fig. 1B). Isolated after only 4 selection cycles, Apt5 was observed to be able to prevent HA docking onto cell receptors. Thus, both DNA and RNA-based aptamers can be employed as inhibitors for influenza viral infectivity and have the potential to not only be detection reagents but also therapeutics.

Francisella tularensis (Tularemia, Cat-A pathogen). Commonly referred to as "rabbit fever", "deer-fly fever" and "market men's disease", Tularemia is often considered to be one of the most infectious bacterial diseases. The bacteria responsible for the disease are known as Francisella tularensis and is endemic to many/all parts of the US. It usually resides in ticks and deer flies, and is transmitted to 
humans through their bite wounds. The bacteria primarily infect and reside in the host's white blood cells, and are able to evade the immune system. At an opportunistic moment, the bacteria will begin its replication cycle and subsequently invade other organs throughout the body. Upon the failure of major human organs, fatality occurs. Its high degree of infectivity is illustrated by observations that as few as 10 organisms are able to cause tularemia in humans. F. tularensis has four subspecies, in which only the tularensis and holarctica are pathogenic to human.

Isolation of DNA aptamers that specifically binds $F$. tularensis was recently reported by Vivekananda and Kiel.[17] The authors utilized nitrocellulose (NC) filter binding for partitioning the bacteria-bound DNA complexes with a total of 10 selection cycles were performed. By using both a sandwich aptamer-linked immobilized sorbent assay and a dot blot analysis, the authors observed that the anti-tularemia ssDNA aptamers, as a cocktail of 25 unique DNA sequences from the selection, were able to specifically bind the antigens from both holarctica and tularensis subspecies. The DNA aptamers also demonstarted high selectivity as they did not bind to either pure chicken albumin or chicken lysozyme. However, the binding sequences and their affinity studies were not reported limiting the further characterization of these aptamers or their use in other sensing platforms.

Ricin Toxin (Cat-B pathogen). Ricin is a toxic protein isolated from the seeds of castor bean plant, Ricinus communis. Ricin is extremely poisonous with reports that $0.2 \mathrm{mg}$ of the toxic protein is sufficient to kill an adult. Since the castor bean plant can easily be cultivated, ricin can be purified and generated without the need of complicated laboratory equipments. The ability to develop an efficient, rapid and accurate sensing system for ricin is thus essential. The ricin protein is comprised of two main domains, namely the A-chain and B-chain. The two domains are simply linked to each other via a disulfide bond. The primary role of the ricin B-chain serves to assist the A-chain to gain entry to targets cells, thus the B-chain is not toxic by itself. However, once inside the cell the A-chain is extremely toxic as it disrupts the protein synthesis process by targeting the ribosomal RNA.

Hesselberth and co-workers have shown RNA aptamers can be selected against the ricin A-chain with high affintiy and specificity.[18] The RNA aptamers were generated via 9 selection cycles using the $\mathrm{NC}$ filter partitioning approach. Upon analysis of the obtained selected aptamers, it was observed that the sequences bear no resemblance to its usual target ribomsal RNA substrate. To understand the necessary conserved region that is responsible for ricin A-chain binding, the aptamer was further minimized to 31nt without any loss in binding affinity. Upon using the Mfold secondary structure prediction software, the short $31 \mathrm{nt}$ aptamer stretch was still able to adopt the putative necessary secondary structure, and exhibited a $\mathrm{K}_{\mathrm{d}}$ of $7.3 \mathrm{nM}$ against the A-chain.

Instead of targeting the ricin A-chain domain, Tang and co-workers have proceeded to purify the whole intact ricin protein from decorticated castor beans. The intact ricin protein was susbequently 
utilized as a target for ssDNA aptamer generation.[19] Anti-ricin DNA aptamers were partioned in two ways, namely through traditional affinity chromatography and capillary electrophoresis (CE). The main advantage of CE is the efficiency needed to generate the high affinity aptamers as generally only $\sim 4$ rounds of selection cycles is required (Bowser refernces would be good here), whereas using traditional affinity chromatography $\sim 8$ or more selection cycles are usually required. Upon completeion of the selection cycles via both approcahes and surveying the binding affinities of individual aptamers from each selection approach, it was observed that the aptamers derived from affinity chromatography possesses $\mathrm{K}_{\mathrm{d}} \mathrm{S}$ in the 105-514 $\mathrm{nM}$ range, whereas CE-derived aptamers have $\mathrm{K}_{\mathrm{d}} \mathrm{S}$ from 58-195 nM. These observations suggest CE-SELEX can increase selection efficiency for higher affinity aptamers.

Trypanosomes-Alder Protozoa (Cat-B pathogen and its simulants). Protozoa are defined as single-celled eukaryotes and are generally considered to be the "smallest of all animals". However, certain parasitic protozoans are extremely infectious and they pose a serious threat to humans. For example, Cryptosporidium parvum is a water-borne protozoan parasite that results in disease manifested through a lengthy period of symptoms such as watery diarrhea, cramps, nausea and anorexia. Another related protozoic parasite that has been garnering a lot of attention lately is the Trypanosomes. Trypanosomes are ubiquitous parasites of numerous hosts and have both threatened and claimed the lives of numerous humans, cattle, and other domesticated animals throughout Africa and South America in recent decades. Trypanosome infections are invariably fatal, and it could take weeks to years for them to exert their pathogenicity depending upon the species and the virulence of individual strains. These blood parasites are the main etiological agents for diseases such as Sleeping sickness (T. brucei) and Chagas's disease (T. cruzi). Although Trypanosomes are not listed in the NIAID priority list, the protozoa are nonetheless highly pathogenic and are considered to be viable simulants for all protozoic parasites.

Goringer and co-workers have been investigating the generation and application of RNA aptamers as potential therapeutic tools against $T$. brucei in recent years,[20,21] and they have successfully generated three distinct classes of RNA aptamers against live T. brucei.[22] It was observed that the RNA aptamers are able to cross link to a single $42 \mathrm{kDa}$ protein located within the flagellar pocket of the $T$. brucei parasites, suggesting the potential binding site for the aptamers. Interestingly, the aptamers were not able to discriminate other strains of Trypanosomes, implying that the aptamers could thus function as general "markers" on the surface for these extracellular parasites. More importantly, they could also function as therapeutic agents against most strains of trypanosomes. The same group has recently generated a 2'- $\mathrm{NH}_{2}$-modified RNA aptamer that binds live trypanosomes with an affinity of $\sim 70$ nM.[23]• It was observed (or hypothesized?) that this aptamer adopts a G-quartet structure upon target binding and due to its structural modifications, it is stable in human serum of $\geq 30 \mathrm{hrs}$. Again, the site of RNA aptamer binding was observed to be in the flagella attachment zone. 
Beside T. brucei, Ulrich and co-workers have recently reported the generation of RNA aptamers against the cell adhesion receptors of $T$. cruzi.[24] The cell adhesion receptors consist of a matrix of host cell proteins which include fibronectin, laminin, thrombospondin and heparin sulfate. Using centrifugation for partitioning the RNA-protozoa complexes, the pool of aptamers generated after 7 selection cycles (via targets displacement) are observed to have an overall $\mathrm{K}_{\mathrm{d}}$ of $\sim 170 \mathrm{nM}$. More importantly, a few of selected aptamers are also observed to be active in vitro inhibitors for the $T$. cruzi invasion process against monkey kidney cells.

Salmonella Typhi (Cat-B pathogen). Besides anthrax dissemination via the mail, the only other documented incident of bioterrorism attack occurred in the state of Oregon in the mid 1980s. Executed by members of the Rajneeshee cult, with the main motive being trying to affect the outcome of a local election, restaurant salad bars were intentionally contaminated with salmonella bacteria. Although no fatalities resulted from this episode, it highlights the fact that these biothreat agents can be easily disseminated to inflict widespread panic and potential financial losses to targeted communities and/or nations. Salmonella are rod-shaped, gram-negative bacteria in which many sub-species exist. In general, salmonella is a foodborne illness. For example, typhoid fever is a serious illness caused by Salmonella typhi. Typhoid fever usually occurs in countries that lack clean water and basic hygienic practices. The bacteria are found in human excrement, and can be easily transmitted via contaminated water or food. Thus their rapid detection and identification is highly desired.[25]

Pili are hollow, hairline structures that enable bacterial attachment. It was observed that when mutations are introduced to the gene that encodes the pili (pils) in S. typhi, their ability to adhere and invade human gastrointestinal epithelial cells in vitro was drastically reduced.[26] This suggests that the structural protein PilS may have important roles in the pathogenesis of S. Typhi in humans. Pan and coworkers have recently reported the generation of RNA aptamers against the type IVB pili of S. Typhi, which is composed largely of polymerized PilS protein.[27] After 8 rounds of selection cycles, the antipili aptamers obtained are observed to bind the PilS protein with a $\mathrm{K}_{\mathrm{d}}$ of $\sim 7 \mathrm{nM}$. In specific, consensus sequences (present eight times in the 14 clones studied) within individual RNA aptamers was AGCG-(X)$\mathrm{GG}$, in which " $\mathrm{X}$ " is any nucleotides. The secondary structure of the consensus sequences, after modeling studies, is anticipated to exist as a "stem-loop".

\section{APTASENSING PLATFORMS AND DETECTION ASSAYS}

Advances in automated protein production techniques has fueled the widespread utility and application of protein-based microarrays.[28] One main drawback of the protein microarray chip is that the immobilized protein may denature through structural rearrangement. To confer structural robustness in the molecular recognition elements suited for the planar microarray sensing platform, aptamers have 
successfully been demonstrated as the next viable option in recent years.[29,30] Ellington and coworkers, have pioneered the design and fabrication of aptamer microarrays,[31-33] in which the group has enabled an automated protocol for aptamer generation.. It is projected that with the augment of automated SELEX selection process, nucleic acid aptamers can now be generated in days, rather than weeks to months.

Instead of a planar platform, suspended encoded beads have gained popularity as of late since the beads can be rapid decoded to render interaction information.[34] Furthermore, the beads can be engineered such that they are easily manipulated by either magnetic forces or electric field. For example, Luminex Corp (Austin, TX; www.luminexcorp.com) has manufactured polystyrene beads which are injected with different ratios of fluorescent dyes. Each bead is thus conferred a unique spectral characteristic, and the beads can be subjected to multiplex interrogation since each interacting event on individual beads can efficiently be sorted via flow cytometry and rapidly decoded. Recently, Luminex beads have been conjugated with various antibodies for sensing biowarfare pathogenic simulants, e.g. plague, anthrax.[35] In addition, aptamers have successfully been used by Porschewski and co-workers as antibody replacements using Luminex beads as platforms.[36] Aside from polystyrene beads, Fan and coworkers have reported the use of dielectric microsphere as aptamer sensing platform.[37] Dielectric microspheres support a series of optical resonance modes at the surface called whispering gallery modes. By attaching anti-thrombin aptamers onto the microspheres, it was observed that the thrombin protein can be detected with a detection limit of $1 \mathrm{NIH}$ unit $/ \mathrm{mL}$.

Depending on their aggregation state, nanoparticles (NP) have been reported to enable different color emission. Taking advantage of this observation, Liu and Lu have described a novel strategy for an aptamer-mediated colorimetric sensor based on the dissolution of a NP aggregate (Fig. 2A).[38]• Initially, the colorimetric aptamer-based sensor is an aggregated complex comprised of AuNPs, aptamers and oligonucleotide linkers. The oligonucleotide sequences utilized in this study are comprised of antiadenosine or anti-cocaine aptamer sequences. Upon the addition of their small molecule targets, the aptamers are able to undergo a structure switching process to enable tight binding to its small molecule target. This results in the displacement of an AuNP-conjugated complementary strand, thus causing the overall AuNP deaggregation process. Consequently, a visible color change ensues due to changes in both the color and extinction of the AuNP..

Based on the same principle, Huang and co-workers have described a colorimetric approach for a protein detection sensing system.[39] In the presence of platelet-derived growth factor (PDGF) protein targets, the aptamer-AuNP system begins to aggregate. Subsequently, changes to both the color and extinction of the AuNP, due to changes in aggregation states, can be monitored in real time (Fig. 2B). The 
color of the AuNP was observed to change from red to purple when low PDGF protein concentration $(<400 \mathrm{nM})$ is added.

Instead of using AuNP, Ellington and co-workers have utilized quantum dots (QD) as platforms for aptamer-based detection.[40] An anti-thrombin aptamer was first hybridized with a short quencher oligonucleotide labeled with a quencher dye, followed by immobilization onto the QDs. Since the quencher dye is in close proximity to the QD, its emission is quenched (Fig. 2C). In the presence of the thrombin target, the aptamer undergoes a structural change to accommodate the thrombin target, displacing the quencher oligonucleotide. The release of the quencher from the QD surface restores the original QD emission profile. Another noted advantage is the QDs have tuneable wavelength emissions. Thus, by attaching different aptamer constructs onto different quantum dots in a heterogeneous solution, multiplex interrogation of numerous target molecules is feasible.

Several elegant bioelectronics sensing systems have been described by Willner and co-workers over the last few years. Of particular interest is a recent aptamer-based bioreadout enhancement process that involves the catalytic enlargement of AuNPs (Fig. 3).[41] Anti-thrombin aptamers were first immobilized onto glass substrate surfaces to capture the thrombin target. An aptamer-based sandwich complex was the formed in the presence of thrombin by the addition of AuNP-labeled aptamers. The AuNP were then catalytically enlarged in a solution mixture containing $\mathrm{HAuCl}_{4}$. By increasing the effective size of the AuNPs in this manner, the AuNP coupled plasmon absorbance at $650 \mathrm{~nm}$ was enhanced and could be efficiently measured. The sensitivity for thrombin detection was observed to be $\sim 20 \mathrm{nM}$, approximately five-fold better than colorimetric detection via organic polymer chromophores.

In another novel extension involving the application of the aptamer displacement detection strategy, Willner and co-workers have developed a label free, reagentless Ion-Sensitive Field Effect Transistor (ISFET) aptamer-based sensing device.[42] Upon binding to adenosine monophosphate (AMP), an AMP aptamer releases a hybridized short complementary oligonucleotide, which in turn affects the charges associated with the gate and alters the source-to-drain current (Fig. 4). This approach demonstrated a sensitivity limit of $5 \times 10^{-5} \mathrm{M}$, requiring only a 4 min response time. The sensing system was also highly specific as other monophosphates, e.g CMP, GMP and TMP, did not result in changes to the ISFET potential. The reported sensitivity represents a 10 -fold enhancement over currently available colorimetric or electrical aptamer-based sensing systems.

Another label-free impedance spectroscopy bioelectronic detection assay was recently reported by Wang and co-workers, in which the binding event is reflected through changes in charges of the electrode's surfaces.[43] Specifically, anti-lysozyme aptamers were first conjugated onto Indium Tin Oxide electrode transducers. Negatively charged $\left[\mathrm{Fe}(\mathrm{CN})_{6}\right]^{3-4-}$ was used as a marker, and in the absence of protein target, the marker is repelled from the surface. However upon protein binding, the negatively 
charged marker is attracted to the electrode surface, subsequently increasing the electron transfer from the marker to the electrode. Besides the detection assay high sensitivity ( $\sim 140$ fmole of lysozyme target in 10 $\mu \mathrm{L}$ sample volume), the assay is also highly specific as addition of non-binding proteins such as thrombin, cytochrome $\mathrm{C}$ and albumin do not affect the electron transfer resistance of $\left[\mathrm{Fe}(\mathrm{CN})_{6}\right]^{3-/ 4-}$.

To enable multiplexed detection via electrochemical aptamer biosensors, Hansen and co-workers have reported the usage of a variety of distinct inorganic QD tracers as aptamer labeling reagents.[44] It was observed that both $\mathrm{Cd}^{2+}$ and $\mathrm{Pb}^{2+}$-based QDs possess excellent coding and amplification features. Hence via the single step displacement assay and subsequent application of electrochemical stripping detection of the QD tracers, the obtained detection sensitivity was observed to be in the sub-pM range (Fig. 5). Although only 2 analytes were used in the study, this approach can be readily expanded for multiplexed target interrogation by encompassing other types of QD tracers.

Most aptamers, such as thrombin-binding ssDNA, usually undergo some form of structural rearrangement (or folding process) upon target binding. Plaxco and co-workers have recently described an electronic aptamer-based (EAB) sensing system in which by tethering a redox probe, e.g. methylene blue, at the end of the aptamer, the resulting folded aptamer-protein complex is able to render the redox probe in close proximity to the Au-surface (Fig. 6).[45,46] - This results in an electron transfer between the redox probes to the $\mathrm{Au}$ surface and thus the resulting electrochemical change indicates a positive binding event. With the ability to rapidly fabricate the aptamers in an array format, a multiplex pathogen sensing aptamer-based microarray chip can be envisioned for their rapid identification and/or characterization.

Tan and co-workers have engineered light-switching excimer probes for rapid and sensitive detection of the PDGF protein.[47]• Labeled with one pyrene at each end of the aptamer, the binding of the protein target brings the two pyrene molecules in close proximity to eachother. The resulting shift in the fluorescence emission from $400 \mathrm{~nm}$ (pyrene monomer) to $485 \mathrm{~nm}$ (pyrene excimer) can be monitored in biological fluids using both steady-state and time-resolved fluorescence measurements (Fig. 7A). Thus, this approach is ideally suited for protein function studies in both intracellular or intercellular environments. Since the described excimer signal approach requires its target molecule to be present to induce structure switching, sample pre-treatment and false signals caused by nucleases degradation and/or nonspecific binding are both eliminated.

Using a similar "bind and read" approach, Li and co-workers have also described the application of FRET-based optical readout when the DNA aptamer undergoes a structural arrangement upon target binding.[48,49] Briefly, a 3-piece DNA sandwich was first formed to bring a fluorophore-labeled DNA oligonucleotide (F-DNA) in close proximity to a quencher oligonucleotide (Q-DNA). In the presence of the target molecule, the unmodified oligonucleotide (MAP), which contains the target binding aptamer 
sequences, will fold to enable tight binding to its target (Fig. 7B). The structural arrangement of the aptamer will result in the displacement of the Q-DNA, which then restores the fluorescence signal.

Aptasensors that are able to channel recognition events into fluorescence changes are of great interest as they are "reagentless" and easy to operate. Taking advantage of allosteric regulation of noncovalent interactions with fluorophores, Stojanovic and Kolpashchikov have recently designed RNA aptamers which contain both recognition and signaling domains, i.e. able to bind to both fluorophores and small molecule targets. [50] When both fluorophores (e.g., malachite green) and small molecule targets (ATP) bind to their respective domains, the overall effect is the generation of an enhanced fluorescence emission. These aptamers are termed "modular aptameric sensors." It was observed through a series of mutation investigations of the communication module stem constructs, that certain aptamer structures, are able to demonstrate fluorescence increases of 3- and 4-fold, respectively, when $1 \mathrm{mM}$ of ATP was added. Hence, should such modular aptameric sensors be expressed in cells, they could be utilized for intracellular imaging.

Along the same "bind and read" aptamer-based fluorescence reporter concept, another novel entry was recently reported by Katilius and co-workers, in which direct bioreadout aptamer-based sensors were designed by strategically positioning fluorescently-modified nucleotides in the sensing aptamer so that the fluorescent nucleotides are in close proximity to the target binding site.[51] The single position in the aptamer for substitution, which can undergo base-stacking changes upon binding to its target, are identified through both computational and structural examinations. The resulting structural rearrangement of the aptamer binding site subsequently effects a fluorescent signal change. For example, based on computational analysis, thymine in position-7 of the anti-thrombin binding aptamer was systematically replaced with fluorescent base analogues. It was observed that specific base analogue such as 6MAP has the greatest effect, in which the relative increase in fluorescence between the bound and unbound states of the aptamers is $\sim 30$-fold.

A novel approach for analyzing the aptamer interaction through a "exonuclease protection" assay was recently reported $\mathrm{Xu}$ and co-workers.[52] Upon binding of the aptamer to its protein target, the protein is able to protect the aptamer from exonuclease I degradation. The remaining aptamer fragment functioned as a linker to join two complementary oligomeric sequences, in which the joined products were subsequently amplified by PCR. Exquisite sensitivities were achieved via this approach as the number of protein targets detected was on the order of $10^{2}$. Furthermore, the assay offers an excellent detection range, encompassing 7 orders of magnitude.

The use of CE to efficiently isolate and/or detect DNA aptamer-protein complexes has been described in recent years.[53,54] These approaches have enabled the aptamer generation process to be more expeditious as the partitioning efficiency of CE is high $\left(\sim 10^{4}\right)$. Hence, very low levels of protein 
targets, to which aptamers are bound, can be isolated. Zhang and co-workers have described the application of CE to isolate the protein-aptamer complex, using PCR amplification of the DNA aptamer to enable high protein detection sensitivity.[55] - It was reported that as few as 6 aptamer molecules are needed for target detection. In addition, Collins and coworkers have also utilized Laser Induced Fluorescence-CE to enable direct detection of sub-nM concentrations of ricin A-chain with fluorescentlylabeled aptamers.[56] When performed in a free-solution assay, a detection limit of $500 \mathrm{pM}$ was achieved, and the presence of other proteins such as BSA, casein and RNA nucleases did not interfere with the aptamer's specificity.

\section{CONCLUSION AND FUTURE DIRECTIONS}

In 2003, it was reported in the Washington Times that documents seized from a high-ranking $\mathrm{Al}$ Qaeda member revealed that a Pakistani microbiologist was recruited by the organization with the intent to manufacture both botulinum toxins and anthrax spores. This clearly illustrates that the threat of bioterrorism, which was once considered to be simply a hypothetical threat, is a clear and present one.[3,57] Primarily due to the ease in the DNA aptamers generation and/or selection process, the low cost of synthesis, and their structural stability, DNA aptamer have evolved to hold great promises as a potential source of sensing ligands for employment in biosensing systems. Although there are still certain limitations to their use, as the general binding affinities of nucleic acid aptamers still do not rival those of monoclonal antibodies, it is evident that the wealth of biorecognition approaches reported in this review hold great promise. Nonetheless, a huge disconnect remains in the successful translation of most of these technologies to be implement in actual product fabrication. With the rapid advances in both aptamer generation against biopathogenic targets and their corresponding bioreadout processes and platforms, it is thus forecasted, with great anticipation, that greater symbiotic relationship with the advent of micro- and nano-engineering will soon enable fieldable, accurate and, perhaps, even implantable multiplexed pathogen aptasensors.

\section{ACKNOWLEDGEMENT}

This work was performed under the auspices of the U.S. Department of Energy by the University of California, Lawrence Livermore National Laboratory, under contract W-7405-Eng-48. N.O.F. acknowledges CMLS for a directorate postdoctoral fellowship and J.B.T. acknowledges NIH Grant AI065359. 


\section{REFERENCES AND RECOMMENDED READING}

Papers of particular interest, published within the annual period of review, have been highlighted as: • of special interest, $\bullet \bullet$ of outstanding interest.

1. Cook-Deegan RM, Berkelman R, Davidson EM, Finder S, Heitman E, Kelley MC, King NM, Moseley

$\mathrm{R}$, Thomas JC, Tilden SJ, et al.: Issues in biosecurity and biosafety. Science 2005, 308:1867-1868.

2. Watkins RE, Eagleson S, Hall RG, Dailey L, Plant AJ: Approaches to the evaluation of outbreak detection methods. BMC Public Health 2006, 6:263.

3. Lim DV, Simpson JM, Kearns EA, Kramer MF: Current and developing technologies for monitoring agents of bioterrorism and biowarfare. Clin Microbiol Rev 2005, 18:583-607.

4. Durodie B: Facing the possibility of bioterrorism. Curr Opin Biotechnol 2004, 15:264-268.

5. Ellington AD, Szostak JW: In vitro selection of RNA molecules that bind specific ligands. Nature 1990, 346:818-822.

6. Tuerk C, Gold L: Systematic evolution of ligands by exponential enrichment: RNA ligands to bacteriophage T4 DNA polymerase. Science 1990, 249:505-510.

7. Bunka DH, Stockley PG: Aptamers come of age - at last. Nat Rev Microbiol 2006, 4:588-596.

8. Jayasena SD: Aptamers: an emerging class of molecules that rival antibodies in diagnostics. Clin Chem 1999, 45:1628-1650.

9. Bruno JG, Kiel JL: In vitro selection of DNA aptamers to anthrax spores with electrochemiluminescence detection. Biosens Bioelectron 1999, 14:457-464.

10. Zhen B, Song YJ, Guo ZB, Wang J, Zhang ML, Yu SY, Yang RF: [In vitro selection and affinity function of the aptamers to Bacillus anthracis spores by SELEX]. Sheng Wu Hua Xue Yu Sheng Wu Wu Li Xue Bao (Shanghai) 2002, 34:635-642.

11. Zuker M: Mfold web server for nucleic acid folding and hybridization prediction. Nucleic Acids Res 2003, 31:3406-3415.

12. Perez DR, Sorrell EM, Donis RO: Avian influenza: an omnipresent pandemic threat. Pediatr Infect Dis J 2005, 24:S208-216, discussion S215.

13. Misono TS, Kumar PK: Selection of RNA aptamers against human influenza virus hemagglutinin using surface plasmon resonance. Anal Biochem 2005, 342:312-317.

14. Gopinath SC, Misono TS, Kawasaki K, Mizuno T, Imai M, Odagiri T, Kumar PK: An RNA aptamer that distinguishes between closely related human influenza viruses and inhibits haemagglutininmediated membrane fusion. J Gen Virol 2006, 87:479-487.

15. Gopinath SC, Sakamaki Y, Kawasaki K, Kumar PK: An efficient RNA aptamer against human influenza B virus hemagglutinin. J Biochem (Tokyo) 2006, 139:837-846. 
16. Jeon SH, Kayhan B, Ben-Yedidia T, Arnon R: A DNA aptamer prevents influenza infection by blocking the receptor binding region of the viral hemagglutinin. Journal of Biological Chemistry 2004, 279:48410-48419.

17. Vivekananda J, Kiel JL: Anti-Francisella tularensis DNA aptamers detect tularemia antigen from different subspecies by Aptamer-Linked Immobilized Sorbent Assay. Lab Invest 2006, 86:610-618.

18. Hesselberth JR, Miller D, Robertus J, Ellington AD: In vitro selection of RNA molecules that inhibit the activity of ricin A-chain. J Biol Chem 2000, 275:4937-4942.

19. Tang J, Xie J, Shao N, Yan Y: The DNA aptamers that specifically recognize ricin toxin are selected by two in vitro selection methods. Electrophoresis 2006, 27:1303-1311.

20. Goringer HU, Homann M, Zacharias M, Adler A: RNA aptamers as potential pharmaceuticals against infections with African trypanosomes. Handb Exp Pharmacol 2006:375-393.

21. Goringer HU, Homann M, Lorger M: In vitro selection of high-affinity nucleic acid ligands to parasite target molecules. Int J Parasitol 2003, 33:1309-1317.

22. Homann M, Goringer HU: Combinatorial selection of high affinity RNA ligands to live African trypanosomes. Nucleic Acids Res 1999, 27:2006-2014.

23. Homann M, Lorger M, Engstler M, Zacharias M, Goringer HU: Serum-stable RNA aptamers to an invariant surface domain of live African trypanosomes. Comb Chem High Throughput Screen 2006, 9:491-499.

24. Ulrich $\mathrm{H}$, Magdesian MH, Alves MJ, Colli W: In vitro selection of RNA aptamers that bind to cell adhesion receptors of Trypanosoma cruzi and inhibit cell invasion. $J$ Biol Chem 2002, 277:2075620762.

25. Goldschmidt MC: The use of biosensor and microarray techniques in the rapid detection and identification of salmonellae. $J A O A C$ Int 2006, 89:530-537.

26. Zhang XL, Tsui IS, Yip CM, Fung AW, Wong DK, Dai X, Yang Y, Hackett J, Morris C: Salmonella enterica serovar typhi uses type IVB pili to enter human intestinal epithelial cells. Infect Immun 2000, 68:3067-3073.

27. Pan Q, Zhang XL, Wu HY, He PW, Wang F, Zhang MS, Hu JM, Xia B, Wu J: Aptamers that preferentially bind type IVB pili and inhibit human monocytic-cell invasion by Salmonella enterica serovar typhi. Antimicrob Agents Chemother 2005, 49:4052-4060.

28. MacBeath G, Schreiber SL: Printing proteins as microarrays for high-throughput function determination. Science 2000, 289:1760-1763.

29. Lee HJ, Wark AW, Li Y, Corn RM: Fabricating RNA microarrays with RNA-DNA surface ligation chemistry. Anal Chem 2005, 77:7832-7837.

30. Stadtherr K, Wolf H, Lindner P: An aptamer-based protein biochip. Anal Chem 2005, 77:34373443. 
31. Cox JC, Ellington AD: Automated selection of anti-protein aptamers. Bioorg Med Chem 2001, 9:2525-2531.

32. Cox JC, Rajendran M, Riedel T, Davidson EA, Sooter LJ, Bayer TS, Schmitz-Brown M, Ellington AD: Automated acquisition of aptamer sequences. Comb Chem High Throughput Screen 2002, 5:289299.

33. Cox JC, Hayhurst A, Hesselberth J, Bayer TS, Georgiou G, Ellington AD: Automated selection of aptamers against protein targets translated in vitro: from gene to aptamer. Nucleic Acids Res 2002, 30:e108.

34. Wilson R, Cossins AR, Spiller DG: Encoded microcarriers for high-throughput multiplexed detection. Angew Chem Int Ed Engl 2006, 45:6104-6117.

35. McBride MT, Masquelier D, Hindson BJ, Makarewicz AJ, Brown S, Burris K, Metz T, Langlois RG, Tsang KW, Bryan R, et al.: Autonomous detection of aerosolized Bacillus anthracis and Yersinia pestis. Anal Chem 2003, 75:5293-5299.

36. Porschewski P, Grattinger MA, Klenzke K, Erpenbach A, Blind MR, Schafer F: Using aptamers as capture reagents in bead-based assay systems for diagnostics and hit identification. J Biomol Screen 2006, 11:773-781.

37. Zhu HY, Suter JD, White IM, Fan XD: Aptamer based microsphere biosensor for thrombin detection. Sensors 2006, 6:785-795.

38. Liu J, Lu Y: Fast colorimetric sensing of adenosine and cocaine based on a general sensor design involving aptamers and nanoparticles. Angew Chem Int Ed Engl 2006, 45:90-94.

39. Huang CC, Huang YF, Cao Z, Tan W, Chang HT: Aptamer-modified gold nanoparticles for colorimetric determination of platelet-derived growth factors and their receptors. Anal Chem 2005, 77:5735-5741.

40. Levy M, Cater SF, Ellington AD: Quantum-dot aptamer beacons for the detection of proteins. Chembiochem 2005, 6:2163-2166.

41. Pavlov V, Xiao Y, Shlyahovsky B, Willner I: Aptamer-functionalized Au nanoparticles for the amplified optical detection of thrombin. J Am Chem Soc 2004, 126:11768-11769.

42. Zayats M, Huang Y, Gill R, Ma CA, Willner I: Label-free and reagentless aptamer-based sensors for small molecules. $J$ Am Chem Soc 2006, 128:13666-13667.

43. Rodriguez MC, Kawde AN, Wang J: Aptamer biosensor for label-free impedance spectroscopy detection of proteins based on recognition-induced switching of the surface charge. Chem Commun (Camb) 2005:4267-4269.

44. Hansen JA, Wang J, Kawde AN, Xiang Y, Gothelf KV, Collins G: Quantum-dot/aptamer-based ultrasensitive multi-analyte electrochemical biosensor. J Am Chem Soc 2006, 128:2228-2229.

45. Xiao Y, Piorek BD, Plaxco KW, Heeger AJ: A reagentless signal-on architecture for electronic, aptamer-based sensors via target-induced strand displacement. J Am Chem Soc 2005, 127:1799017991. 
46. Xiao Y, Lubin AA, Heeger AJ, Plaxco KW: Label-free electronic detection of thrombin in blood serum by using an aptamer-based sensor. Angew Chem Int Ed Engl 2005, 44:5456-5459.

47. Yang CJ, Jockusch S, Vicens M, Turro NJ, Tan W: Light-switching excimer probes for rapid protein monitoring in complex biological fluids. Proc Natl Acad Sci U S A 2005, 102:17278-17283.

48. Nutiu R, Li Y: Structure-switching signaling aptamers. J Am Chem Soc 2003, 125:4771-4778.

49. Rupcich N, Chiuman W, Nutiu R, Mei S, Flora KK, Li Y, Brennan JD: Quenching of fluorophorelabeled DNA oligonucleotides by divalent metal ions: implications for selection, design, and applications of signaling aptamers and signaling deoxyribozymes. $J$ Am Chem Soc 2006, 128:780790.

50. Stojanovic MN, Kolpashchikov DM: Modular aptameric sensors. J Am Chem Soc 2004, 126:92669270.

51. Katilius E, Katiliene Z, Woodbury NW: Signaling aptamers created using fluorescent nucleotide analogues. Anal Chem 2006, 78:6484-6489.

52. Wang XL, Li F, Su YH, Sun X, Li XB, Schluesener HJ, Tang F, Xu SQ: Ultrasensitive detection of protein using an aptamer-based exonuclease protection assay. Anal Chem 2004, 76:5605-5610.

53. Berezovski M, Musheev M, Drabovich A, Krylov SN: Non-SELEX selection of aptamers. $J$ Am Chem Soc 2006, 128:1410-1411.

54. Mosing RK, Mendonsa SD, Bowser MT: Capillary electrophoresis-SELEX selection of aptamers with affinity for HIV-1 reverse transcriptase. Anal Chem 2005, 77:6107-6112.

55. Zhang H, Wang Z, Li XF, Le XC: Ultrasensitive detection of proteins by amplification of affinity aptamers. Angew Chem Int Ed Engl 2006, 45:1576-1580.

56. Haes AJ, Giordano BC, Collins GE: Aptamer-based detection and quantitative analysis of ricin using affinity probe capillary electrophoresis. Anal Chem 2006, 78:3758-3764.

57. Lane HC, Montagne JL, Fauci AS: Bioterrorism: a clear and present danger. Nat Med 2001, 7:1271-1273. 


\section{Annotations:}

- 3. An exhaustive review paper which provides readers an excellent understanding of the various advances in monitoring biowarfare agents.

- 12. The article describes the generation of RNA aptamers against whole viruses. The RNA aptamer analyzed have remarkable affinity (as it is $\sim 15$-fold higher that monoclonal antibody) and selectivity (able to differentiate the same protein (HA) form different viral strains. The aptamer is also observed to be able to prevent the binding of the virus to targeted cells in vitro.

- 20. This article describes the application of two approaches, namely CE and affinity chromatography, to generate DNA aptamers against whole Ricin protein. Through the CE approach, it was observed that the aptamers generated have higher affinities against the Ricin target and is faster to generate.

$\bullet \bullet 24$. This article describes the generation of 2'- $\mathrm{NH}_{2}$ modified RNA aptamers against live Trypanosomes protozoa. The aptamers are observed to be stable in human serum for over 30 hours, highlighting their structural robustness and their potential application as MREs in sensing system.

- 35. An overall concise, yet comprehensive, review paper detailing the latest advances in encoded particles. Enhancing information derived from these encoded particles would greatly facilitate the multiplex targets detection and characterization.

- 39. The article describes the application of AuNP-aggregate, via aptamer and complementary oligonucleotides, for small molecule detection. The de-aggregation process, due to displacement of "linking" complementary oligonucleotides, results in visible colorimetric changes.

- 47. The article describes the development of electronic aptamer-based (EAB) sensing system based on the displacement of complementary oligonucleotides. The attachment of redox probe at the end of aptamer will then be able to allow electron transfer.

- 49. The article describes the novel design of pyrene end-labeled aptamer that is able to shift its fluorescence emission, upon target binding, through excimer formation. The excimer's long fluorescence lifetime suppresses the background signals, making the assay adaptable for most test mediums.

- 53. The article describes the strategic incorporation of fluorescent nucleotide analogues to existing signaling aptamer such that fluorescence enhancement up to 30-fold can be achieved upon target binding.

- 57. The article describes the application of CE to first isolate target bound reporter DNA aptamer. Subsequent PCR amplification of reporter aptamer enables ultrasensitive determination of target protein 


\section{FIGURES:}

Figure 1. [A] Secondary structures of studied DNA sequences against $b$. anthracis spores, as predicted by the Mfold program.[9] (i) Full-length 78nt Apt1 binding aptamer, (ii) a minimized 32nt Apt2 binding aptamer from a different sequence, (iii) further minimization of Apt2 to a 17nt Apt3 aptamer abolished its binding affinities, showing that the predicted single stranded region is also important for spore binding. [B] Secondary structures, aspredicted by the Mfold program,[10] of (i) RNA sequences against the HA of Human influenza A and (ii) DNA sequences against the HA (aa 91-261) of the Influenza A virus.

$[\mathrm{A}]$

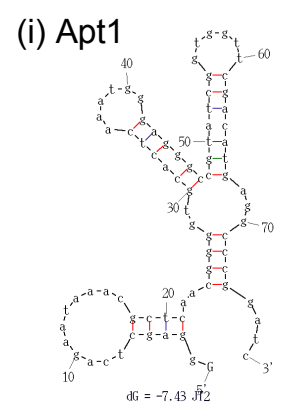

[B]

(i) Apt4

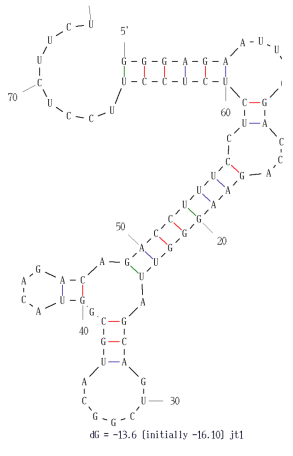

(ii) Apt 2

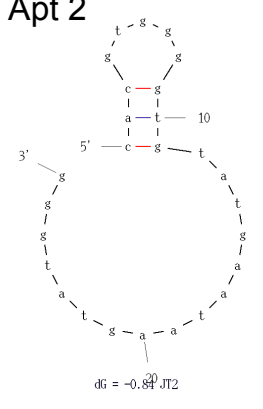

(iii) Apt $3_{t}{ }^{-8}-s$

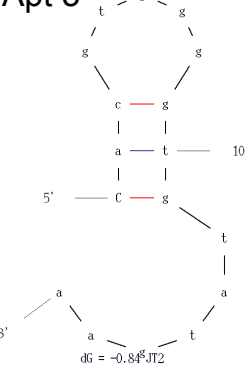

(ii) Apt 5

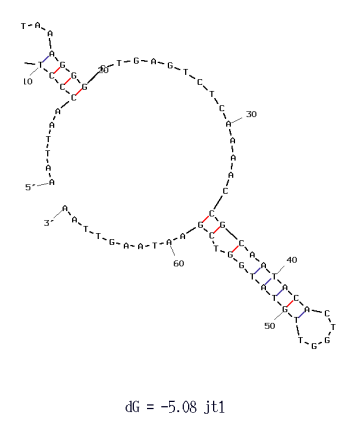


Figure 2. [A] Colorimetric detection of cocaine based on cocaine-induced disassembly of NP aggregates linked by a cocaine aptamer. Folding of the anti-cocaine aptamer displaces the AuNP-conjugated reporter oligonucleotide, thus resulting in the deaggregation of the AuNP.[39•] [B] With increasing PDGF protein target concentrations, different degrees of AuNP aggregation are obtained, . resulting in different colorimetric changes, e.g. red to purple.[40] [C] (i) QD-aptamer beacon for the detection of thrombin target proteins. (ii) Binding of protein targets displaces the quencher oligonucleotide and enhances the fluorescence of the quantum dot. [41]

$[\mathrm{A}]$

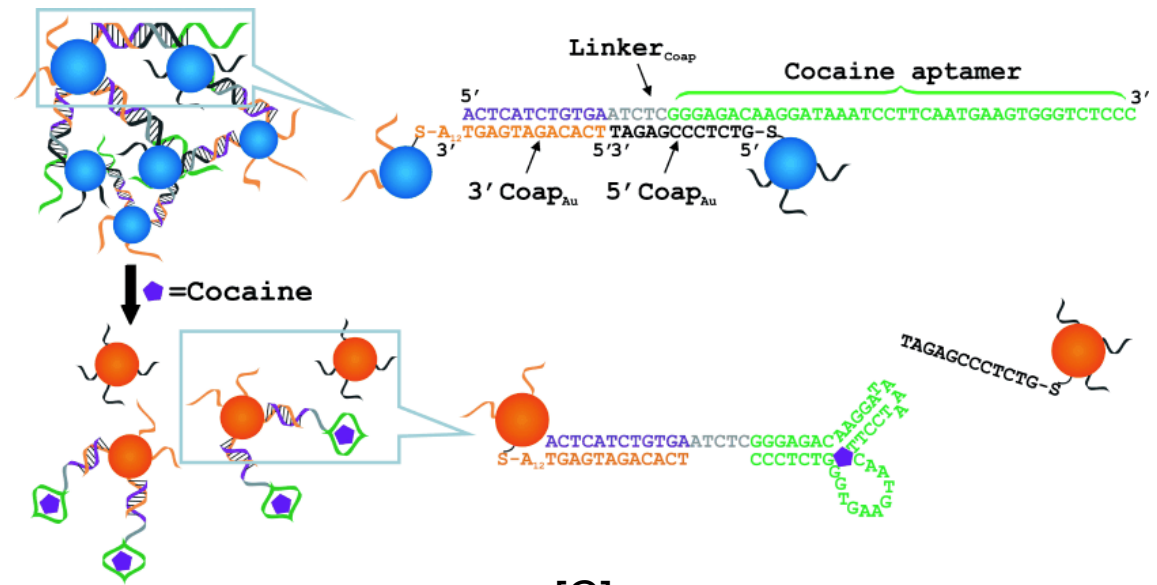

$[\mathrm{B}]$

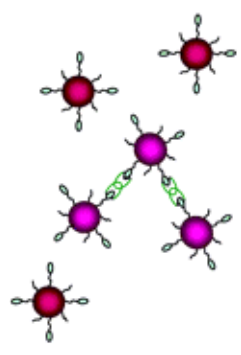

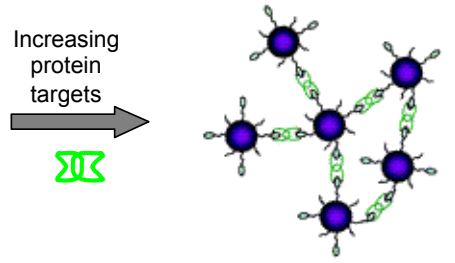

(i)

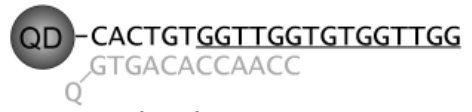

two-piece beacon construct

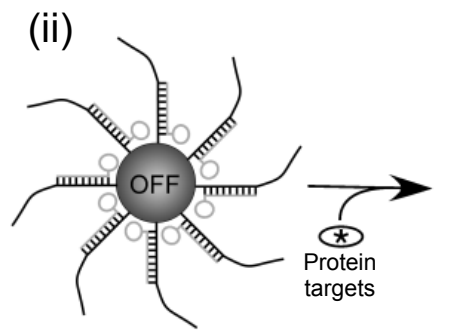

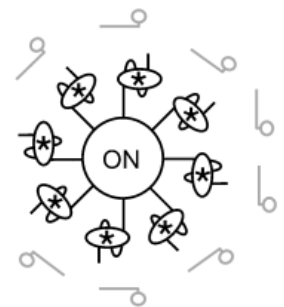


Figure 3. Amplified detection of thrombin on surfaces by the catalytic enlargement of AuNPs functionalized with anti-thrombin aptamers . [43]

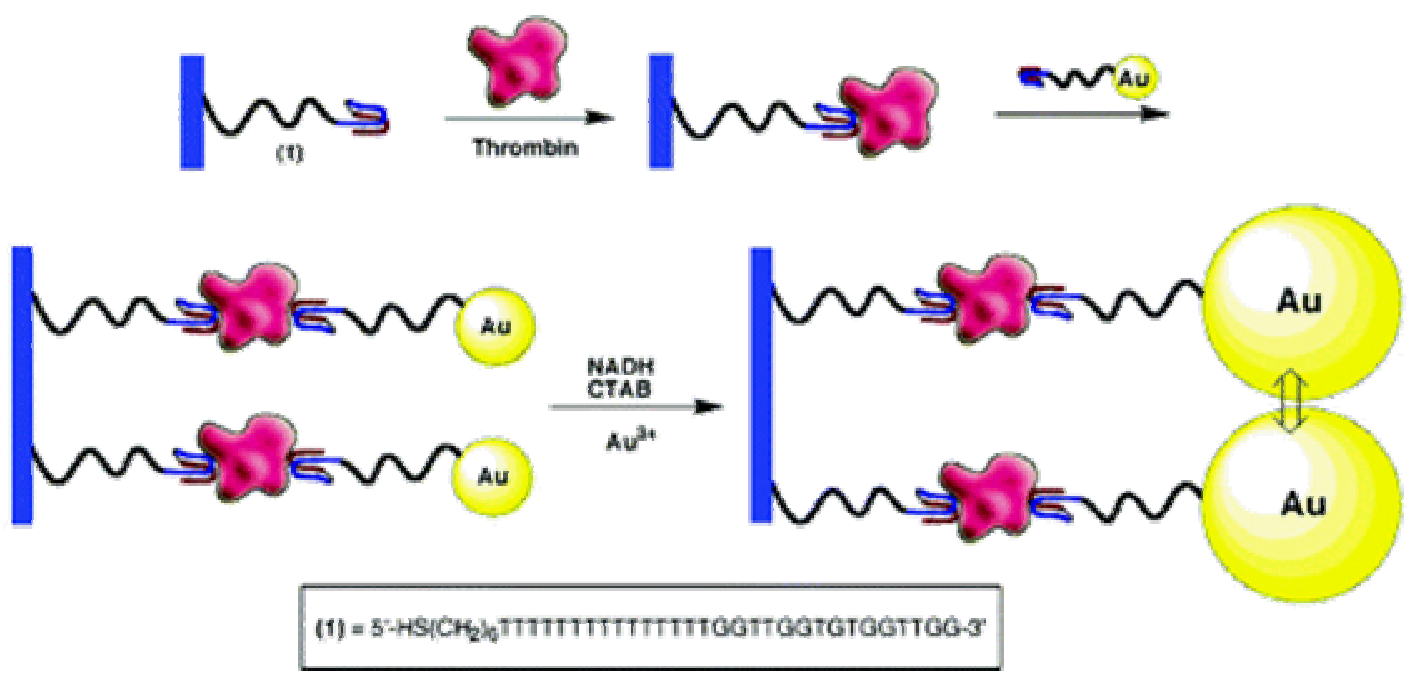

Figure 4. Schematic of a label-free, reagentless ISFET aptasensor device for small molecule (adenosine) detection. [44] The release of the short complementary oligonucleotide will then affect the charges associated with the gate, thus altering the source-to-drain current.

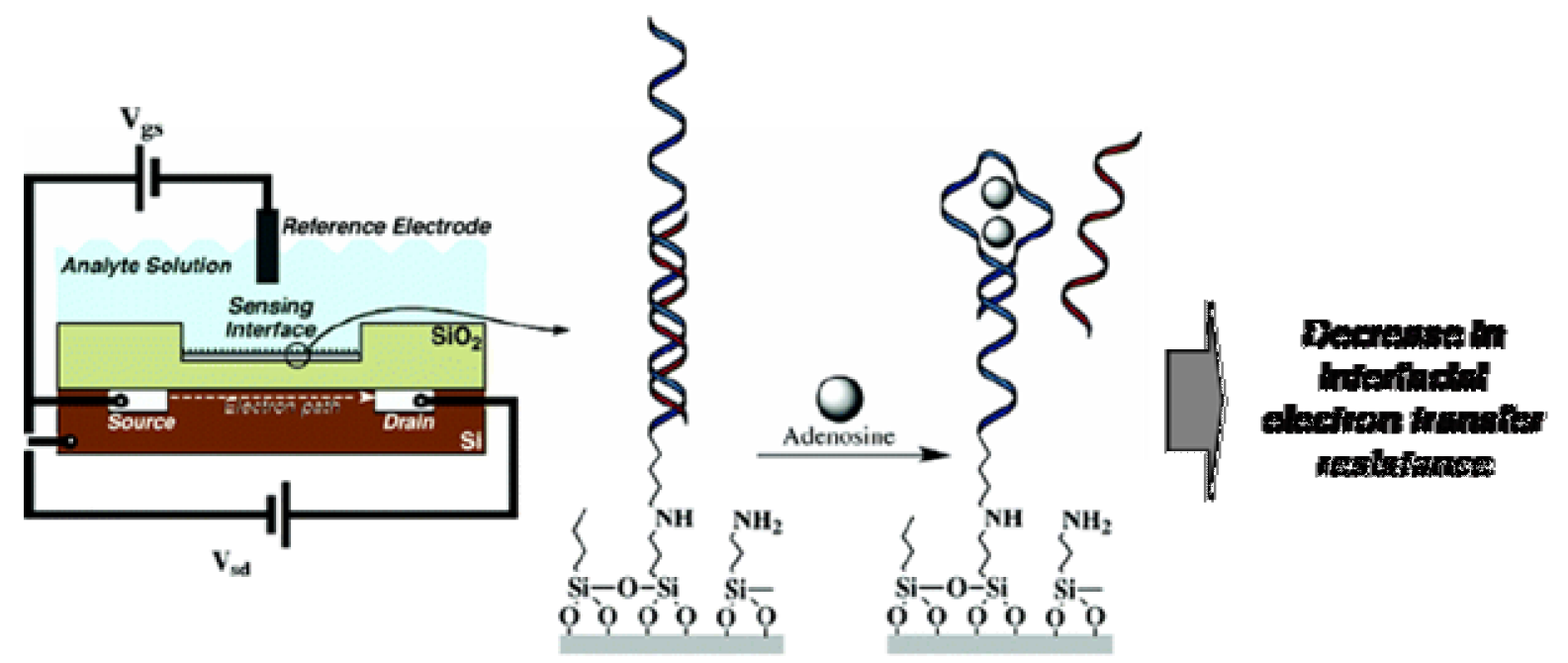


Figure 5. [A] A dual analyte aptamer-based biosensor involving the displacement of QD-tagged proteins by the target analytes.[46] Briefly, the QDs on the displaced tagged proteins are subjected to electrochemical-stripping detection at a coated glassy carbon electrode. [B] Schematic illustration of label-free protein detection via monitoring changes in electron transfer through negatively charged $\left[\mathrm{Fe}(\mathrm{CN})_{6}\right]^{3-/ 4-}$ markers. [45]

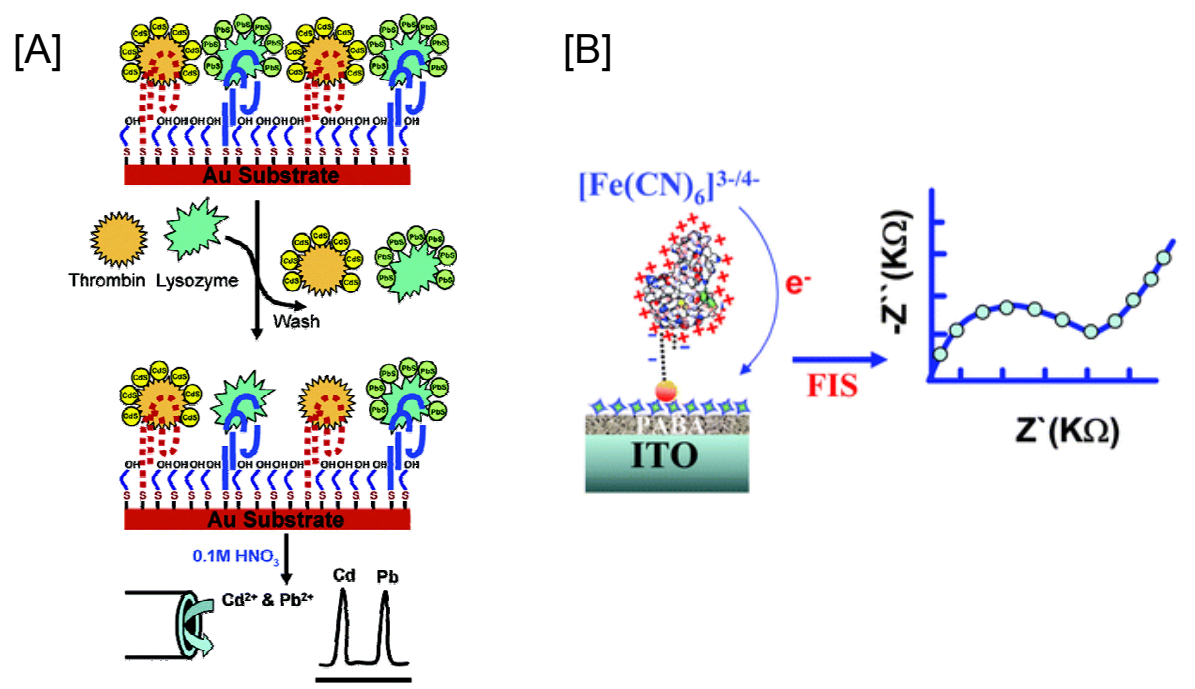

Figure 6. Schematic illustration of the electronic aptamer-based (EAB) sensing system. [47•]
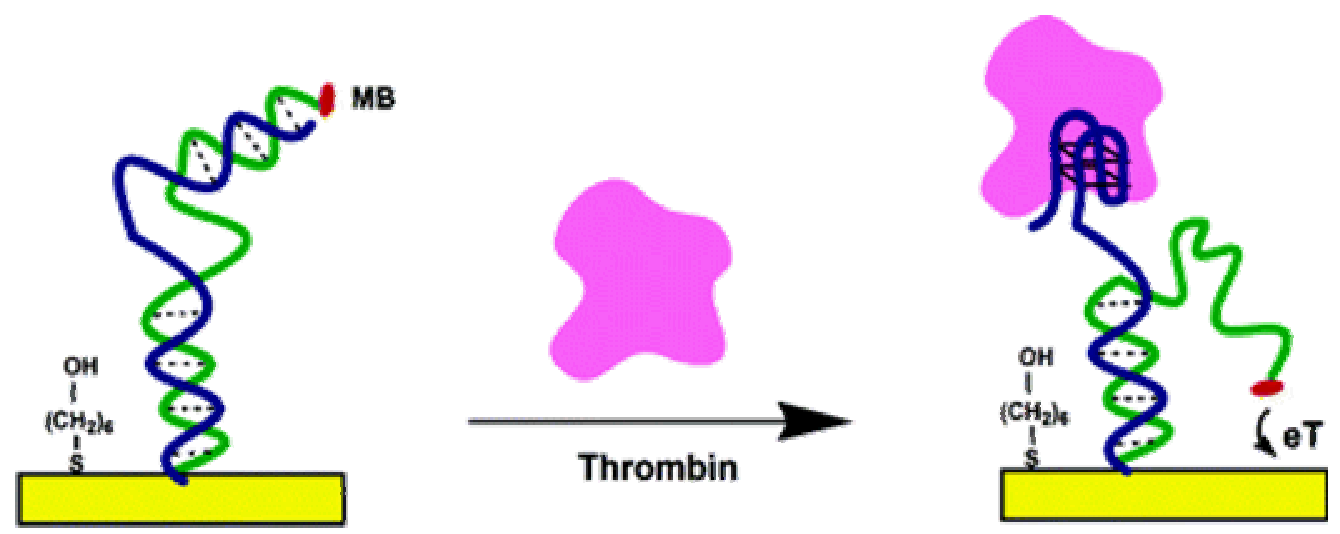
Figure 7. [A] Schematic illustration of the pyrene end-labeled excimer to detect the PDGF protein. The anti-PDGF aptamer is labeled with pyrene molecules at both ends. The open structure of the aptamer ensures that the pyrene molecules remain as monomers (emission peaks at $\sim 400 \mathrm{~nm}$ ). Upon binding to PDGF protein target, the aptamer adapts a close conformation, thus bringing the two pyrene molecules in close proximity. Consequently, pyrene excimers form and green light $(\sim 480 \mathrm{~nm})$ is emitted after photoexcitation.[49•] [B] A schematic illustration of the FRET-based structure-switching signaling aptamer assay.[ref] Again, the structural arrangement of the aptamer upon target biding will result in the displacement of the Q-DNA, which then restores the fluorescence signal stemming from the F-DNA.
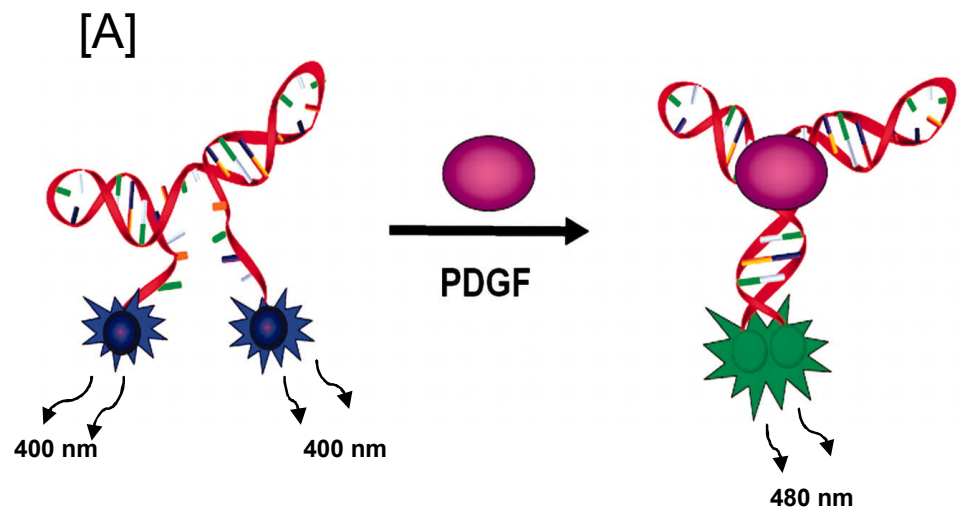

[B]

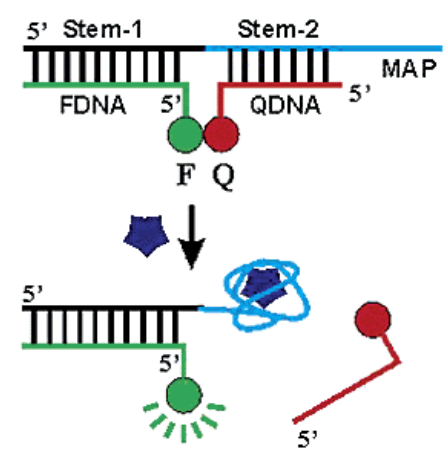

